\title{
Complete prefrontal lobe isolation surgery for recurrent epilepsy: A case report
}

\author{
SHAOYA YIN ${ }^{1}$, WEIPENG JIN ${ }^{2}$, QINGYUN LI ${ }^{3}$, MEI FENG ${ }^{4}$, KEKE FENG ${ }^{1}$, \\ HUI SHAO ${ }^{1}$, XUEQING ZHANG $^{4}$ and SHIMIN WANG ${ }^{3}$ \\ ${ }^{1}$ Department of Neurosurgery, Tianjin Huanhu Hospital, Tianjin 300060; \\ ${ }^{2}$ Department of Neurosurgery, Second Affiliated Hospital, Tianjin Medical University, Tianjin 300211; \\ Departments of ${ }^{3}$ Neurology and ${ }^{4}$ Nerve Electrophysiology, Tianjin Huanhu Hospital, Tianjin 300060, P.R. China
}

Received February 7, 2015; Accepted March 22, 2016

DOI: $10.3892 / e t m .2016 .3714$

\begin{abstract}
Epileptogenic focus resection is less effective for the treatment of frontal lobe epilepsy compared with temporal lobe epilepsy. However, there is currently a lack of effective therapeutic options for patients with frontal lobe epilepsy who are unsuitable for epileptogenic focus resection (such patients with epileptogenic foci in one frontal lobe in which the precise epileptic foci cannot be determined), or who experience recurrent epilepsy following epileptogenic focus resection. The present study reports a patient with frontal lobe epilepsy who underwent successful frontal lobe isolation surgery following a previous unsuccessful epileptogenic focus resection surgery. To ensure complete isolation of the prefrontal lobe, the surgery included division of the anterior commissure and the anterior part of the corpus callosum. The patient was followed-up for 16 months. Although the follow-up electroencephalogram presented a number of sharp waves on the affected side, the patient did not experience any seizures. The results suggest that prefrontal lobe isolation is an effective method of treating frontal lobe epilepsy, as division of the anterior commissure and the anterior part of the corpus callosum ensures disconnection of the prefrontal lobe from other regions of the brain.
\end{abstract}

\section{Introduction}

Epileptogenic focus resection is the primary treatment option for refractory frontal lobe epilepsy; however, it is less effective than epileptogenic focus resection for temporal epilepsy (1). Patients with postoperative recurrence of frontal lobe epilepsy or a high risk of postoperative recurrence, patients with epileptogenic foci primarily in one frontal lobe, or patients

Correspondence to: Dr Shaoya Yin, Department of Neurosurgery, Tianjin Huanhu Hospital, 122 Qixiangtai Road, Hexi, Tianjin 300060, P.R. China

E-mail: yinshaoya2014@163.com

Key words: frontal lobe epilepsy, isolation surgery, incision surgery, anterior commissure, corpus callosum with epileptogenic foci in one frontal lobe in which the precise area of foci cannot be determined, often undergo prefrontal lobe resection with preservation of the motor area. However, removal of large areas of brain tissue may result in substantial postoperative complications, and thus prolonged hospitalization and high costs (2).

Previous outcomes following hemispheric and posterior quadrantic disconnection surgery demonstrate that disconnection surgery results in the preservation of the majority of the biologically active brain tissues in the epileptogenic zone, and the isolation of the epileptogenic zone from other cerebral cortical regions and central structures $(3,4)$. These procedures have similar rates of seizure control to the corresponding anatomical resection procedures, but are associated with reduced surgical injury and fewer postoperative complications. Disconnection surgery is currently used with increasing frequency for the treatment of intractable epilepsy when the epileptogenic foci are in one hemisphere, or in the posterior part of a hemisphere (5-8). The principle of 'more incision, less excision' is becoming increasingly accepted by surgeons.

Numerous studies of disconnection surgery in patients with unilateral temporal lobe or temporal-parietal-occipital lobe epilepsy have been reported (5-7,9-13). However, to the best of our knowledge, no studies of disconnection surgery for frontal lobe epilepsy have been reported. Based on the successful implementation of hemispheric disconnection and posterior quadrantic disconnection surgery for epileptogenic foci in other regions $(11,13)$, the concepts of epileptogenic focus isolation' and 'more incision, less excision' were applied in the current case report. The report describes the treatment of a patient with frontal lobe epilepsy with recurrence following resection of epileptogenic foci. Following complete frontal lobe isolation surgery, the patient had no further seizures during the 16 months of the follow-up period. In the present study, the surgical anatomy, surgical technique and indications for frontal lobe isolation surgery are discussed.

\section{Case report}

A 17-year-old female was hospitalized on March 11, 2013 (Department of Neurosurgery, Tianjin Huanhu Hospital, Tianjin, China). The patient had a 14-year history of epileptic 
seizures. At 3 years of age the patient was experiencing 7-8 seizures per day, which involved right eyelid twitching, associated with fear and suddenly holding onto people. The patient was diagnosed with epilepsy secondary to nodular sclerosis at the Department of Epilepsy, Tianjin Children's Hospital (Tianjin, China) and was treated orally with topiramate, followed by carbamazepine and valproate (dose and supplier unknown). Each of these medications was only effective during the first 20 days of administration. The present study was approved by the Ethics Committee of Tianjin Huanhu Hospital. Written informed consent was obtained from the father of the patient.

In 2008, the patient underwent resection of left frontal lobe epileptogenic foci at the Epilepsy Center, Yuquan Hospital (Beijing, China), but this did not reduce the frequency of the seizures. The patient subsequently developed psychiatric symptoms and personality changes (the patient became stubborn, greedy and capricious). Prior to admission to the Department of Neurosurgery, Tianjin Huanhu Hospital, in March 2013, the patient was administered oxcarbazepine, levetiracetam and clonazepam sequentially, but the seizures were not reduced. The patients seizures were preceded by sudden fear and holding onto people, followed by absence and secondary tonic-clonic seizures. The patient experienced psychiatric symptoms, irritability and personality changes. Physical examination showed normal growth and development, an unkempt appearance and a bandaged right hand, which had been burned when it came into contact with a object during a seizure. A Wechsler Memory Scale (14) was used to evaluate the patient's memory prior to surgery, and it was found that the patient's memory was poor (Wechsler score, 78). During the examination, the patient was uncooperative, unable to concentrate and hyperactive; normal limb muscle strength and muscle tension was observed, with no pathological tendon reflexes.

Magnetic resonance imaging (MRI; Magnetom Avanto 1.5T; Siemens Healthcare GmbH, Erlangen, Germany) showed evidence of the previous left frontal craniotomy, an irregular prefrontal lobe, expanded subarachnoid space, and abnormal signals in the cortex and subcortex of the frontal orbital gyrus and gyrus rectus (Fig. 1A and B). Vigilance-controlled electroencephalogram (EEG; Bio-logic System Corporation, Lake County, IL, USA) changes were monitored by long-range video EEG. Ten seizures characterized by excessive movements were observed during a 24 -h period. While resting, the patient suddenly showed signs of discomfort and reduced blinking, and did not respond to being called. Her head and body twisted from the supine position into the prone position, associated with involuntary pedaling movements of the lower limbs. The longest episode lasted $80 \mathrm{sec}$. During this activity, the EEG showed frequent irregular long-range low/moderate-amplitude slow waves at 2-3 cycles/sec, originating in the left temporal region and involving all the left-sided leads. There were also isolated moderate-amplitude sharp waves in the left anterior temporal region. During seizures, paroxysmal low-amplitude fast waves at 30 cycles/sec with gradually increasing amplitude were observed in all leads, in particular in the bilateral prefrontal temporal regions. The wave rate gradually slowed to $\sim 8$ cycles/sec. Moderate-amplitude sharp waves and sharp-slow waves were detected in the posterior frontal region, in particular on the right side. The left-sided leads showed irregular paroxysmal moderate/high-amplitude slow waves at 2-3 cycles/sec. The wave amplitudes were very high in the left temporal regions. Low/moderate-amplitude alpha activity at 10 cycles/sec was observed in the parieto-occipital leads (Fig. 1C and D). These results suggested a diagnosis of frontal lobe epilepsy. Originally, it was thought that epileptogenic focus resection or expanded focus resection would be unsuccessful. However, complete left prefrontal lobe isolation surgery was performed on March 18, 2013, including anatomical incision of the prefrontal lobe, the anterior part of the corpus callosum and the anterior commissure.

Under general anesthesia using propofol $(2.5 \mathrm{mg} / \mathrm{kg}$; Aztra Zeneca, London, UK) fentanyl citrate $(0.01 \mu \mathrm{g} / \mathrm{kg})$, cosatracurium $(0.6 \mathrm{mg} / \mathrm{kg})$ and Remifentanyl $(2.4 \mathrm{mg} / \mathrm{ml})$ (all purchased from Jiangsu Hengrui Medicine Co., Ltd., Linayungang, China), the patient was positioned supine, and her head was fixed with a Mayfield head frame. Craniotomy was performed at the site of the previous frontotemporal scalp incision, through the left frontal bone at the midline. The dura was cut in a petal shape, and the superior frontal gyrus and middle frontal gyrus had areas of gliosis with a yellow appearance and solid texture. The surgical procedure was performed as follows: i) White matter at the bottom of the middle part of the superior frontal sulcus was isolated and incised. There was a nodule visible beneath the ventricular ependyma at the anterolateral part of the interventricular foramen of the left lateral ventricle. The nodule was solid and was colored yellow and gray, with a poorly defined border with poor vascular supply. There was visible gliosis at the edge of the nodule. The abnormal tissue was removed and sent for pathological examination (4.5 $\mu \mathrm{m}$ tissue size) using formalin-fixing (Tianjin Bodi Cemical Co., Ltd., Tiajnin, China) and hematoxylin and eosin staining (Sigma-Aldrich, St. Louis, MO, USA). Arachnoid from the longitudinal fissure was observed in the medial part of the gray matter. Arachnoid from the skull base, the A1 segment of the anterior cerebral artery and the optic chiasm were observed after the gray matter was incised. ii) An incision was made from the lateral end of the incision previously described, along the frontal horn of the lateral ventricle through the ependyma, white matter and gray matter, to the lateral cleft and the anterior part of the circular sulcus of the insula. The subependymal incision was extended downwards until the bone of the anterior skull base was visible beneath the arachnoid. iii) The middle of the superior frontal gyrus was incised transversely from the superior frontal sulcus in the midline to the arachnoid of the longitudinal fissure, and then directly downwards. The medial parts of the superior frontal gyrus and cingulate gyrus were incised towards the corpus callosum. The pericallosal artery was observed, but the arachnoid was left intact to protect the artery. The fibers of the anterior part of the corpus callosum were transected anteriorly until this incision joined the incision made previously in the anterior part of the interventricular foramen. iv) The cortices of the middle frontal gyrus and inferior frontal gyrus were incised transversely outwards, from the incision in the superior frontal sulcus to the anterior end of the lateral cleft. The incision was made deep to the left lateral ventricle, end extended through the cortex, white matter and ependyma. v) The corpus callosum and anterior commissure were incised. The longitudinal fissure 

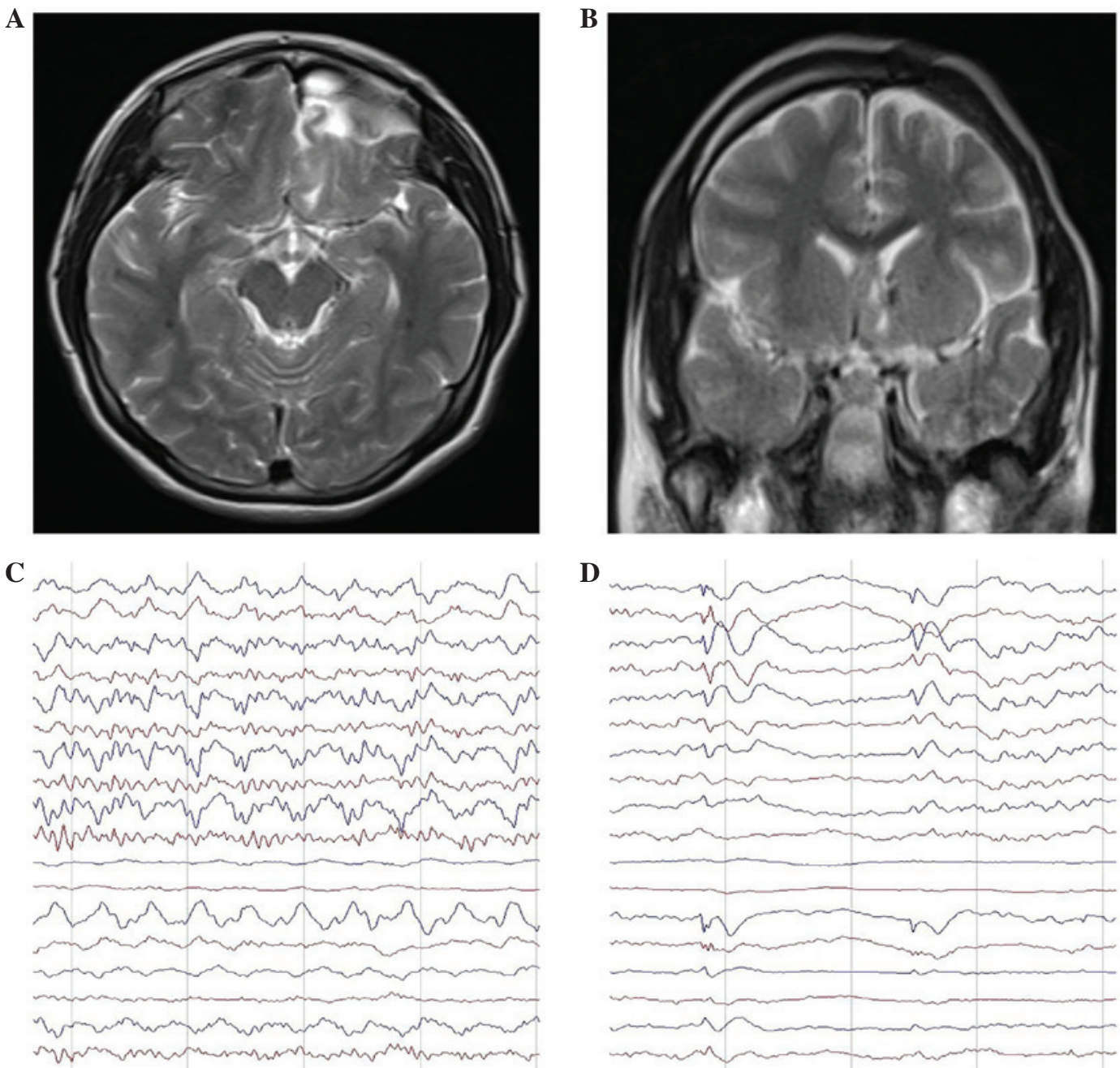

Figure 1. Magnetic resonance imagine (MRI) and electroencephalogram (EEG) changes on admission into hospital. (A and B) T2-weighted MRI images, showing the previous left frontal craniotomy, an irregular prefrontal lobe, enlarged subarachnoid space, abnormal signals in the cortex and subcortex, mixed abnormal signals in the orbital gyrus and gyrus rectus of the frontal lobe, and numerous non-enhanced lesions. (C and D) While in a resting state, the patient suddenly experienced reduced blinking, did not respond to being called and had increased motor activity. (C) During seizures, there were paroxysmal low-amplitude fast waves at $30 \mathrm{cycles} / \mathrm{sec}$ in all the leads, in particular in the bilateral prefrontal temporal regions. The amplitude gradually increased and the wave rate gradually decreased to $\sim 8$ cycles/sec. (D) The interictal EEG showed frequent irregular long-range low/moderate-amplitude slow waves at 2-3 cycles/sec, originating in the left temporal region and involving all the left-sided leads. There were also isolated moderate-amplitude slow sharp waves in the left anterior temporal region.

was incised anteriorly to expose the white corpus callosum. The corpus callosum was incised strictly along the midline between two pericallosal arteries using a microscopic suction device (Sonastar-FS-1000-RF; Misonix, Inc., Farmingdale, NY, USA). In the deep region, a cavity of the septum pellucidum was visible. The incision of the corpus callosum was extended forwards and backwards until 2/3 of the body of the corpus callosum was divided. Anteriorly, the genu and rostrum of the corpus callosum were cut. After entering the cavity of the septum pellucidum, the anterior commissure was divided in the midline until the superior cistern and optic chiasm were observed. vi) The cerebral ventricle and surgical cavity were washed with warm saline. The dura were carefully sutured, and the bone was reset. No surgical drainages tubes were used. The scalp was sutured closed in layers.

When performing the surgery, the skull base, arachnoid of the longitudinal fissure, and arterial and venous circulation of the frontal lobe was left intact to ensure survival of the isolated brain tissue.
The patient received intravenous sodium valporate $(0.5 \mathrm{~g}$ twice daily; Sanofi Pharmaceutical Co., Ltd., Hangzhou, China) and dexamethasone (5 mg every $12 \mathrm{~h}$; Wonder Pharmaceutical Co.,Ltd., Shanghai, China) for 3 days after surgery. Postoperative recovery was uneventful, and the patient was discharged 10 days after surgery. Pathological examination of the resected nodule showed a pilocytic astrocytoma. The patient was treated with oral oxcarbazepine, and no seizures were detected during 16 months of follow-up. Three months after surgery, MRI showed complete isolation of the left frontal lobe and division of the corpus callosum and anterior commissure (Fig. 2). A repeat EEG examination showed a number of sharp waves in the left prefrontal temporal region, with no spread to the right side. At 1 year after surgery, the patient's Wechsler score improved to 89.

\section{Discussion}

Intractable localized epilepsy originates in the frontal lobe in $\sim 20 \%$ of cases. Frontal lobe epilepsy is less frequent, but more 

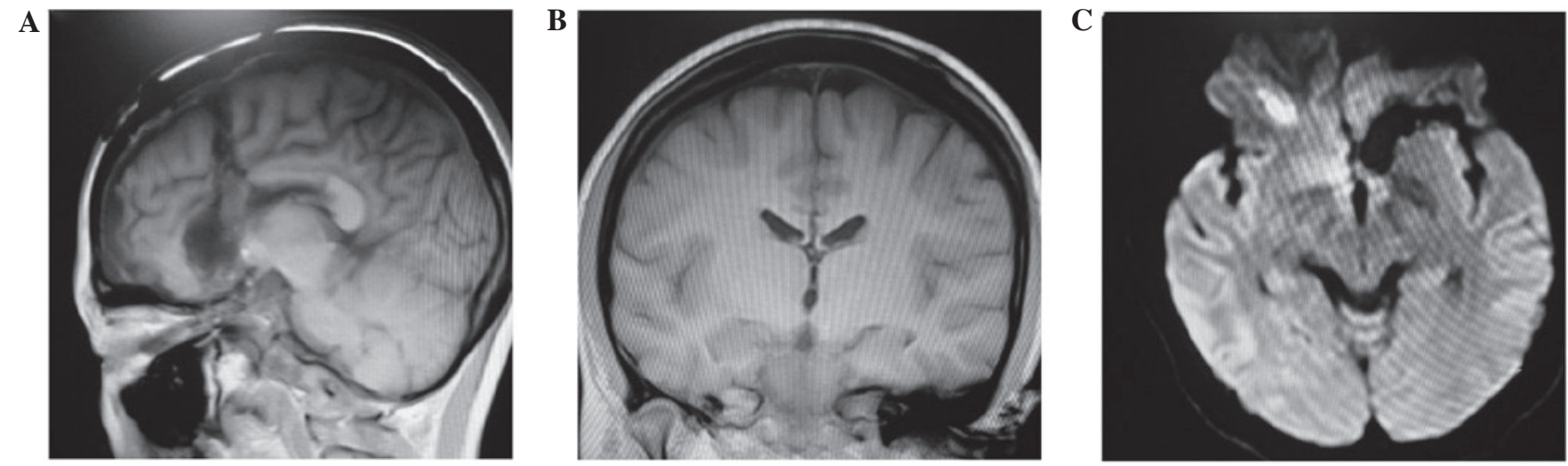

Figure 2. Postoperative magnetic resonance imaging; (A) sagittal, (B) coronal and (C) axial views. The left prefrontal lobe, corpus callosum, and anterior commissure were completely divided, and the left frontal lobe was completely isolated. (A and B) Corpus callosum and (C) anterior commissure were incised strictly along the midline.
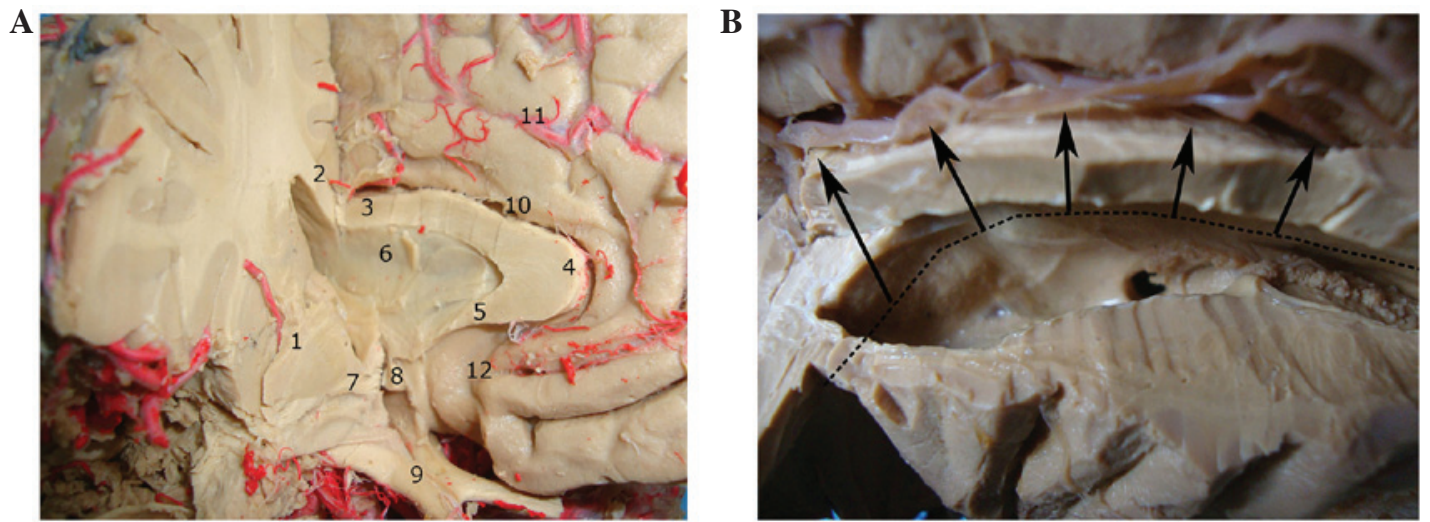

Figure 3. Surgical views. (A) Associations between the midline structures around the right third ventricle; the body, genu and rostrum of the corpus callosum were resected along the midline. After entering the cavity of the septum pellucidum, the anterior commissure was observed. It is important to protect the anterior part of the third ventricle and the optic chiasm. (B) Incision in the medial and ventral aspects of the left frontal lobe. The long black line indicated the location of the cut; the black arrows indicate the direction of the incision. The anterior incision should be anterolateral to the interventricular foramen. The bidirectional black arrow indicates the frontal horn of the cerebral ventricle and the anterior sulcus of the insula. 1, Basal ganglia; 2, corpus callosum body; 3 , corpus callosum body, 4 , corpus callosum knee; 5 , corpus callosum mouth; 6 , septum pellucidum; 7 , anterior commissure; 8 , anterior commissure; 9 , optic chiasma; 10, cingulate gyrus; 11, anterior cerebral artery branch; 12, endplate pool.

problematic, than temporal lobe epilepsy, and patients with frontal lobe epilepsy typically experience several seizures per day (15). The surgery for frontal lobe epilepsy has poor outcomes compared with surgery for temporal lobe epilepsy; therefore, the surgical management of frontal lobe epilepsy is challenging (16).

Epileptogenic focus resection remains the primary surgical procedure for the treatment of frontal lobe epilepsy. However, a previous study demonstrated that the rate of remission following surgery for frontal lobe epilepsy was $80 \%$ in patients with epileptogenic foci, and $45 \%$ in patients without (16). Furthermore, the postoperative outcomes were improved in patients with lateral frontal lobe epilepsy compared with those in patients with medial frontal lobe epilepsy (16). If there are extensive epileptogenic foci in one frontal lobe, if epileptogenic foci can be localized to the frontal lobe but the exact location cannot be determined, or if there is recurrence of seizures following epileptogenic focus resection, surgeons may perform prefrontal lobe resection with retention of the motor area. This procedure may result in the resection of a large quantity of brain tissue and a relatively high risk of postoperative complications, resulting in prolonged hospitalization and high costs (2).

In patients with diffuse lesions affecting both hemispheres, or with epileptogenic foci in functional areas that cannot be resected, division of the corpus callosum, transection of multiple areas, or cortical thermal burn therapy may be beneficial (17). These procedures disrupt the fiber connections between cortical columns, but not the projection fibers from the cortex to the subcortical centers (18). However, they are considered palliative as they may reduce seizure frequency or severity, but do not stop seizure activity (19). A previous study observed that hemispheric and posterior quadrantic disconnection achieved the same rates of seizure control as epileptogenic focus resection in patients with intractable epilepsy (20). Disconnection is achieved by surgical incision to achieve isolation of the epileptogenic foci (21). Such isolation surgery disrupts connections within and between hemispheres, and the projection fibers between the cortex and the subcortical center. The epileptogenic zone retains its biological activity, but is electrophysiologically disconnected from other brain regions, so that the pathways of epileptiform discharges are interrupted (22). 
A

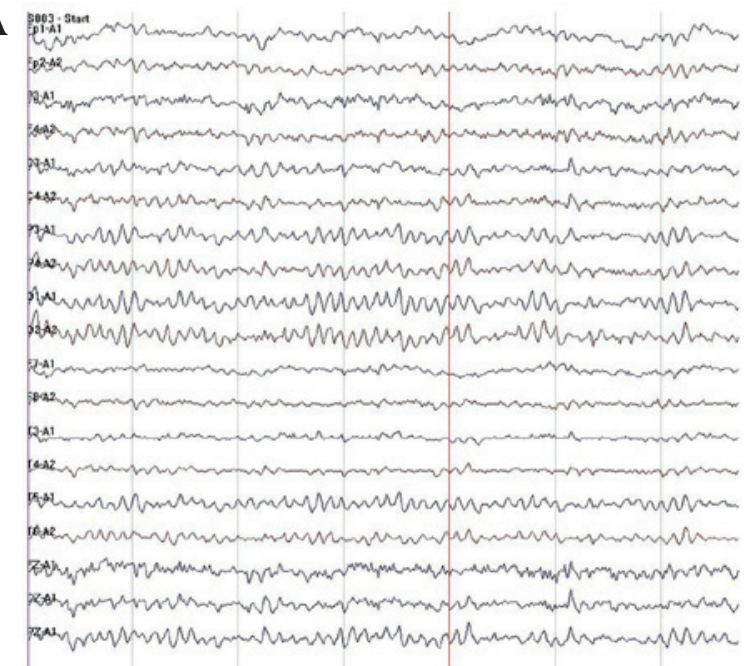

B

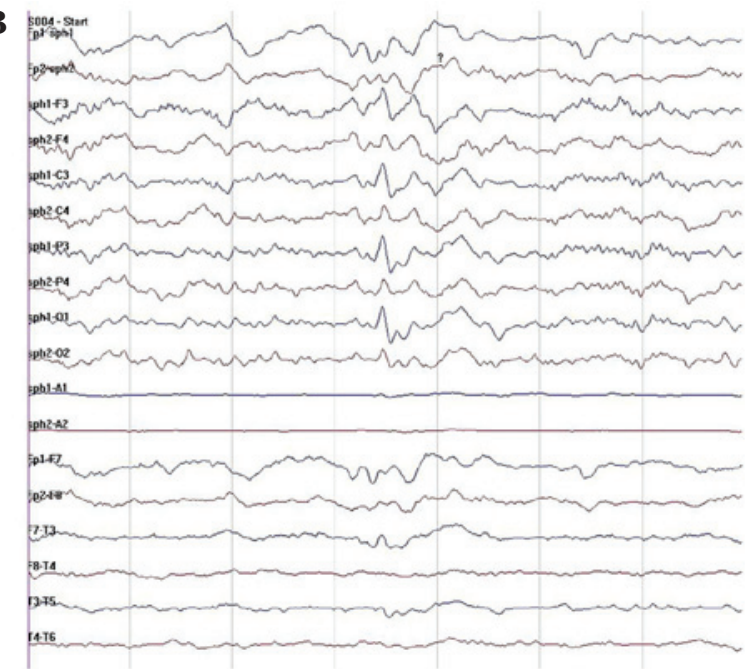

Figure 4. Scalp electroencephalogram following surgery. (A) Background alpha wave activity was detected at 10 cycles/sec with normal voltage, bilateral symmetry, good adjustment and amplitude modulation. (B) There were also intermittent irregular low/moderate amplitude slow waves in the left prefrontal leads, with some sharp waves.

Hemispheric disconnection or posterior quadrantic disconnection surgery preserves the biological activity of the epileptogenic brain tissue, and isolates the epileptogenic zone from other brain regions, the thalamus and basal ganglia (13). Disconnection surgery is becoming increasingly popular for the treatment of intractable epilepsy in patients with extensive epileptogenic foci in one cerebral hemisphere or in the posterior part of a cerebral hemisphere. The concept of 'more incision, less excision' is gaining increasing acceptance; the majority of isolation procedures involve hemispheric disconnection, and increasing numbers of posterior quadrantic disconnections are being reported (22). Temporal lobe incision and hypothalamic hamartoma incision have also been reported (8). For frontal lobe epilepsy, surgery that partially transects the frontal cortex and subcortical white matter without entering the cerebral ventricles has been reported (23); this transects the nerve fibers of the subcortical epileptogenic foci, but does not result in frontal lobe isolation. To the best of our knowledge, no previous studies have reported frontal lobe isolation surgery. The patient in the present study underwent complete prefrontal lobe isolation surgery, and the surgical methods are described in detail in order to encourage standardization of surgical techniques to help with future comparisons of postoperative outcomes.

It is important to completely disconnect the anatomical and functional (neurophysiological) contact between the target region (epileptogenic zone) and other regions to completely isolate the epileptogenic zone, and prevent transmission and amplification of epileptiform discharges (24). To achieve this, the surgeon must have the appropriate knowledge and experience of microdissection techniques. The incision near the interventricular foramen may easily injure the column of the fornix (Fig. 3A and B), which may cause postoperative memory impairment; incomplete division may result in incomplete postoperative seizure control. In the patient in the current study, a curved incision was made in the ependyma anterior and lateral to the interventricular foramen, as far as the skull arachnoid and avoiding the column of the fornix. The anterior part of the corpus callosum and the anterior commissure were also divided. This avoided injury to the column of the fornix, and ensured complete isolation of the frontal lobe.

In the present study, the patient's memory improved postoperatively. Although a number of sharp waves were detected on EEG following surgery, the patient's seizures arrested, suggesting that the epileptogenic zone was fully isolated (Fig. 4B). Similar to posterior quadrantic disconnection, frontal lobe isolation surgery involves the incision of the dorsolateral cortex, followed by incision of the dorsolateral white matter, cerebral ventricle, ventromedial white matter and ventromedial cortex (22). The dorsolateral cortical incision extends from the middle and posterior parts of the superior frontal gyrus, obliquely beneath the surface of the brain to the anterior part of the lateral cleft, and to the anterior part of the circular sulcus of the insula (in the dominant hemisphere, the posterior part of the inferior frontal gyrus should be left intact) (25). The remainder of the procedure is performed as described above, resulting in complete frontal lobe isolation. Postoperative MRI showed division of the anterior part of the corpus callosum and the anterior commissure, with complete isolation of the frontal lobe.

In conclusion, the current study demonstrated that prefrontal lobe isolation surgery may be an effective method of treating frontal lobe epilepsy; however, this conclusion requires confirmation by further studies. The results suggest that frontal lobe epilepsy that has recurred following epileptogenic focus resection, or refractory frontal lobe epilepsy without structural foci detected by MRI, may benefit from complete prefrontal lobe isolation surgery.

\section{Acknowledgements}

The authors thank Professor Zhang Yuqin, Professor Yan Xiaoling and Professor Chen Jun for their technical assistance. The study was supported by the Science and Technology Foundation of Tianjin Health and Family Planning Commission (grant no. 2014KG116). 


\section{References}

1. Mu J, Rampp S, Carrette E, Roessler K, Sommer B, Schmitt FC, De TX, Hamer H, Boon P, Pauli E, et al: Clinical relevance of source location in frontal lobe epilepsy and prediction of postoperative long-term outcome. Seizure 23: 553-559, 2014.

2. Stone JJ, Reynolds MR and Leuthardt EC: Transient hemispatial neglect after surgical resection of a right frontal lobe mass. World Neurosurg 76: 361.e7-e10, 2011.

3. Yin SY, Feng KK, Feng M, Zhang XQ and Zhang YQ: Posterior quadrantic disconnection maintains the activity of isolated temporal-parietal-occipital nerve tissue: Neuroprotective measures in the surgical treatment of epilepsy. Neural Regen Res 9: 447-448, 2014.

4. Lee YJ, Kim EH, Yum MS, Lee JK, Hong S and Ko TS: Long-term outcomes of hemispheric disconnection in pediatric patients with intractable epilepsy. J Clin Neurol 10: 101-107, 2014.

5. Bulteau C, Otsuki T and Delalande O: Epilepsy surgery for hemispheric syndromes in infants: Hemimegalencepahly and hemispheric cortical dysplasia. Brain Dev 35: 742-747, 2013.

6. Dorfer C, Czech T, Dressler A, Gröppel G, Mühlebner-Fahrngruber A, Novak K, Reinprecht A, Reiter-Fink E, Traub-Weidinger T and Feucht M: Vertical perithalamic hemispherotomy: A single-center experience in 40 pediatric patients with epilepsy. Epilepsia 54: 1905-1912, 2013.

7. Kovanda TJ, Rey-Dios R, Travnicek J and Cohen-Gadol AA: Modified periinsular hemispherotomy: Operative anatomy and technical nuances. J Neurosurg Pediatr 13: 332-338, 2014.

8. Massager N, Tugendhaft P, Depondt C, Coppens T, Drogba L, Benmebarek N, De Witte O, Van Bogaert P and Legros B: Long-term outcome of surgical disconnection of the epileptic zone as an alternative to resection for nonlesional mesial temporal epilepsy. J Neurol Neurosurg Psychiatry 84: 1378-1383, 2013.

9. Guan YG, Luan GM and Zhou J: Hemispherotomy for children with intractable epilepsy. Zhong Hua Shen Jing Wai Ke Za Zhi 28: 994-997, 2012 (In Chinese).

10. Su CD, Chang PF and Yu L: Clinical analysis of disconnection surgery of posterior part of the cerebrum for one case of refractory epilepsy. Li Ti Ding Xiang He Gong Neng Xing Shen Jing Wai Ke Za Zhi 25: 247-250, 2012 (In Chinese).

11. Sugano H, Nakanishi H, Nakajima M, Higo T, Iimura $Y$, Tanaka K, Hosozawa M, Niijima S and Arai H: Posterior quadrant disconnection surgery for Sturge-Weber syndrome. Epilepsia 55: 683-689, 2014.

12. Yin SY, Feng KK, Yue W, Feng M, Wang SM and Zhang XQ: Hemisphere disconnection in the treatment of intractable epilepsy. Zhong Hua Shen Jing Wai Ke Za Zhi 29: 714-718, 2013 (In Chinese).
13. Yin SY, Feng M, Li QY, Zhang XG, Yue W, Zhao A, Liu QJ and Zhang YG: Posterior quadrantic disconnection for temporal-parietal-occipital epilepsy in two cases and review of the literature. Zhong Hua Shen Jing Wai Ke Za Zhi 29: 1235-1236, 2013 (In Chinese).

14. Kan R, Watabe M, Takahashi R, Kaneko Y, Miyamoto Y and Niwa S: Comparison of IMP-single photon emission computed tomography findings to Wechsler Intelligence Scale and Benton Visual Memory Scale. Psychiatry Clin Neurosci 49: S225-S227, 1995.

15. Fuentes A and Smith ML: Patterns of verbal learning and memory in children with intractable temporal lobe or frontal lobe epilepsy. Epilepsy Behav 53: 58-65, 2015.

16. Garcia PA and Laxer KD: Lateral Frontal Lobe Epilepsies. Bleasel AF: Medial Frontal Lobe Epilepsy. In: Epilepsy Surgery. Lüders HO and Comair YG (eds). 2nd edition. Lippincott Williams and Wilkins, Philadelphia, PA, pp111-134, 2001.

17. Yin SY: Nerve fiber disconnection treatment for refractory epilepsy. Yi Xue Zong Shu 20: 828-832, 2014 (In Chinese).

18. Peters M, Oeltze S, Seminowicz D, Steinmetz H, Koeneke S and Jäncke L: Division of the corpus callosum into subregions. Brain Cogn 50: 62-72, 2002.

19. Unterberger I, Bauer R, Walser G and Bauer G: Corpus callosum and epilepsies. Seizure 37: 55-60, 2016.

20. Lew SM, Matthews AE, Hartman AL and Haranhalli N; Post-Hemispherectomy Hydrocephalus Workgroup: Posthemispherectomy hydrocephalus: Results of a comprehensive, multiinstitutional review. Epilepsia 54: 383-389, 2013.

21. Massager N, Tugendhaft P, Depondt C, Coppens T, Drogba L, Benmebarek N, De Witte O, Van BP and Legros B: Long-term outcome of surgical disconnection of the epileptic zone as an alternative to resection for nonlesional mesial temporal epilepsy. J Neurol Neurosurg Psychiatry 84: 1378-1383, 2013.

22. Nooraine JR, Shiva K, Iyer RB, Rao RM and Raghavendra S: Posterior quadrant disconnection for refractory epilepsy: A case series. Ann Indian Acad Neurol 117: 392-397, 2014.

23. Guo XD, Wang BH, Wu YZ, Liu MH, Lu WF, Hao WM, Wang ZH, Zhao G and Yang JG: Frontal lobe disconnection for intractable frontal lobe epilepsy of normal imageology. Zhong Hua Shen Jing Wai Ke Za Zhi 29: 512-515, 2013 (In Chinese).

24. Kim DL, Osburn LL and Cohen-Gadol AA: A novel method for confirmation of hemispheric disconnection during hemispherotomy surgery. Pediatr Neurosurg 46: 71-75, 2010.

25. Zhu HT, Zhu JL, Zhao TZ, Wu Y, Liu HY, Wu T, Yang L, Zou YJ, Zhang $R$ and Zheng G: Alteration of interictal brain activity in patients with temporal lobe epilepsy in the left dominant hemisphere: a resting-state MEG study. BioMed Res Int 2014: 171487, 2014. 